Kong. Res. J. 2(1) : 15-17, 2015

ISSN 2349-2694

Kongunadu Arts and Science College, Coimbatore.

\title{
STARCH MEDIATED SYNTHESIS OF HYDROXYAPATITE NANOPARTICLE FOR BIOMEDICAL APPLICATIONS
}

\author{
Indira, $\mathrm{J}^{*}$. \\ Department of Chemistry, Vellalar College for Women (Autonomous), Erode-12 \\ *E.mail: jayabal.indira@gmail.com
}

\begin{abstract}
Hydroxyapatite (HAP) nanoparticles with uniform morphologies and controllable size have been synthesized by template directed method. The environment and eco-friendly polysaccharide soluble starch is used as a template to regulate size and shape of the nanoparticles synthesized. Structural and morphological properties of as-synthesized hydroxyapatite nanoparticles have been examined through the techniques like Fourier Transform Infrared Spectroscopy (FT-IR), X-ray Diffraction (XRD) and Scanning Electron Microscopy(SEM), respectively. The results indicate that the obtained particles are uniform discrete spherical nanoparticles. The average size of the hydroxyapatite nanoparticles were ranged from 45 to $60 \mathrm{~nm}$.
\end{abstract}

Keywords: Hydroxyapatite, Ecofriendly, starch, template, biomedical

\section{INTRODUCTION}

Hydroxyapatite $\left[\mathrm{Ca}_{10}\left(\mathrm{PO}_{4}\right)_{6}(\mathrm{OH})_{2}\right]$ is the principal inorganic constituent of bones and teeth (Dorozhkin et al., 2002). It is widely applied in biomedical engineering as bone substitutes, bone cavity filler, bone cement, coating for implant due to its good biocompatibility, excellent ability to form chemical bond with living bone tissue (i.e., bioactivity), and suitable osteoconductivity (Hench et al.,1998 and Dorozhkin et al., 2002). Considering the copious application of hydroxyapatite in biomedical field, various synthesis techniques have been developed. These include mechanochemical synthesis (Liou et al., 2003), combustion preparation (Hench et al., 1994), and wet chemistry such as direct precipitation from aqueous solution (Raman et al., 2002), sol-gel procedure (Hwang et al., 1999; Bezzi et al., 2003), and hydrothermal synthesis (Yan et al., 2001). The size and shape of hydroxyapatite alter the applicability of this material in various biomedical fields. In this perspective, controlling the size and shape of HAP nanoparticles is a fascinating area of research in recent trends. Complemently, template assisted synthesis method is proved to be a convenient method to control the size and morphology of nanoparticles (Gopi et al., 2012). Moreover, an increasing awareness towards green chemistry and other biological processes has led to the development of an eco-friendly approach for the synthesis of HAP nanoparticles. The use of environmentally benign and renewable materials like soluble starch offers numerous benefits of ecofriendliness and compatibility for pharmaceutical and biomedical applications. In this outlook, herein we report synthesis of hydroxyapatite nanoparticles using starch as template by green synthesis route. The addition of starch of various concentrations how can alter the purity, crystallinity, size and shape of HAP nanoparticles were also discussed.

\section{MATERIALS AND METHODS}

Calcium nitrate tetrahydrate $\left(\mathrm{CaNO}_{3} \cdot 4 \mathrm{H}_{2} \mathrm{O}\right)$ and phosphoric acid $\left(\mathrm{H}_{3} \mathrm{PO}_{4}\right)$ are used the precursors for calcium and phosphate respectively. Aqueous ammonia and soluble starch are the other chemicals in analar grade. In a typical experimental procedure, $0.05 \mathrm{M}$ of $\mathrm{CaNO}_{3} .4 \mathrm{H}_{2} \mathrm{O}$ was mixed with $0.01 \mathrm{wt}$. \% of starch, and the solution was stirred for about $1 \mathrm{~h}$ to ensure the co-operative interaction and the self assembly process was completed. At the same time, $0.03 \mathrm{M}$ of $\mathrm{H}_{3} \mathrm{PO}_{4}$ was added slowly to the above mixture and the stirring was extended to about $16 \mathrm{~h}$. The $\mathrm{pH}$ of the solution was maintained at 9 by using aqueous ammonia.

A milky white precipitate was obtained which is kept in an oven for $24 \mathrm{~h}$ at a temperature to $80^{\circ} \mathrm{C}$. The precipitate is washed with water and ethanol to remove the insoluble precursors and other organic residues. Followed by, the precipitate is dried in an oven for $40^{\circ} \mathrm{C}$. Then it was calcined at $600^{\circ} \mathrm{C}$ for $6 \mathrm{~h}$ to remove the starch then sintered at $900^{\circ} \mathrm{C}$ for $2 \mathrm{~h}$ in a muffle furnace to obtain nanohydroxyapatite powders. The experiment is repeated for the other two different concentration of starch $(0.03 \mathrm{wt} \%$ and $0.05 \mathrm{wt}$ \%) also to compare the effect of its addition on the purity, crystallinity and morphology of HAP.

To characterize the synthesized nanohydroxyapatite, Fourier transform infrared spectra were recorded using Nicolet 380 FT-IR 
spectrophotometer over the range from $4000-400$ $\mathrm{cm}^{-1}$ with a number of scans 32 and resolution 4 $\mathrm{cm}^{-1}$. For this, small amount of HAP powders were blended with $\mathrm{KBr}$ and then pressed into discs for the measurement.

The phase composition and the crystallinity of the HAP powders were determined by X-ray diffraction (XRD) Bruker D-8 Advance-Germany Spectrometer, with $\mathrm{CuK} \alpha$ radiation $\lambda=1.5406 \AA$ generated at $35 \mathrm{kV}$ and $25 \mathrm{~mA}$. Data were collected over the $2 \theta$ range $20-60^{\circ}$ with a step size of $0.010^{\circ}$ and a count time of $0.2 \mathrm{~s}$. The morphology of the nano-HAP powders was examined by scanning electron microscopic (JSM 840A Scanning Microscope, JEOL-Japan.) technique.

\section{RESULTS AND DISCUSSION}

\subsection{Fourier Transform Infra red Spectroscopic Studies}

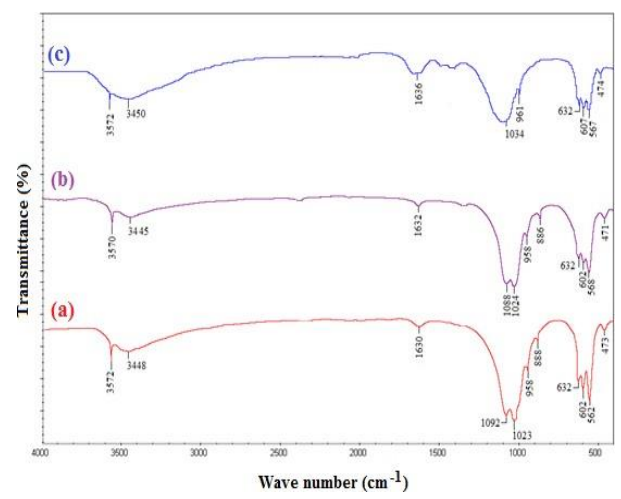

Fig. 1. FTIR spectra of HAP nanoparticles synthesized by template method using starch at three different concentration (a) 0.01 wt.\% (b) 0.03 wt.\% (c) 0.05 wt. \%.

Fig. 1(a-c) shows the FT-IR spectra of HAP nanoparticles synthesized by template method using aqueous starch at three different concentrations. From the fig. 1 (a-c), it is evident that the formation of apatite in all the cases by the observed fundamental vibrational modes of the phosphate peaks at around $1092 \& 1023 \mathrm{~cm}^{-1}\left(\mathrm{v}_{3}\right), 602 \& 562$ $\mathrm{cm}^{-1}\left(\mathrm{v}_{4}\right), 473 \mathrm{~cm}^{-1}\left(\mathrm{v}_{2}\right)$ and $958 \mathrm{~cm}^{-1}\left(\mathrm{v}_{1}\right)$ respectively. The peaks at $3572 \mathrm{~cm}^{-1}$ and $632 \mathrm{~cm}^{-1}$ corresponding to the stretching and bending vibration of the hydroxyl $\left(\mathrm{OH}^{-}\right)$group and are considered as the characteristic peaks of stoichimetric hydroxyapatite. Apart from that the peaks observed at around 3448 $\mathrm{cm}^{-1}$ and $1630 \mathrm{~cm}^{-1}$ is due to the stretching and bending modes of adsorbed water. These findings were well coincide with earlier results (Gopi et al., 2010). There are no impurities formed as evident from the FTIR results.

\subsection{X-ray Diffraction Studies}

The X-ray diffraction patterns for the HAP nanoparticles synthesized by template method using starch at various concentrations are shown in Fig. 2 (a-c).

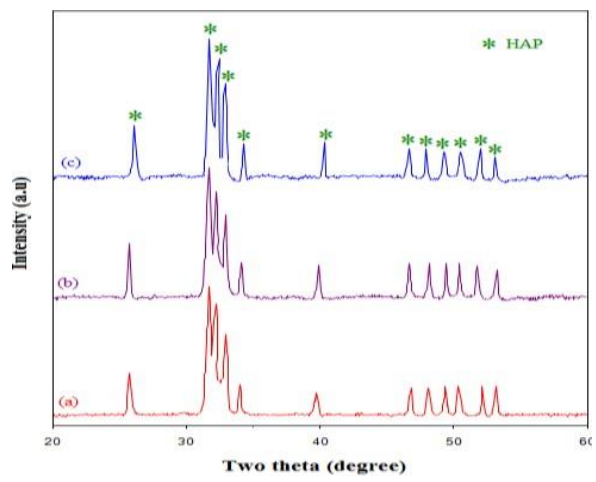

Fig. 2 XRD pattern of HAP nanoparticles synthesized by aqueous starch as template at three different concentrations (a) 0.01 wt.\% (b) 0.03 wt.\% (c) 0.05 wt.\%.

All the peaks in the XRD pattern shown in Fig. $2(a-c)$ are attributed to stoichimetric HAP which is well consistent with the ICDD No.09-0432 and no other calcium phosphate peaks were detected (Gopi et al., 2012). On increasing the starch concentration, the intensity of the XRD peaks shown in Fig. 2(a-c) seems to be increased thus suggesting the increased crystallinity of the HAP particles.

\subsection{Scanning Electron Microscopic Studies}

Fig. 3 (a-c) shows the SEM images of HAP nanoparticles synthesized by template directed method using aqueous starch at three different concentrations.

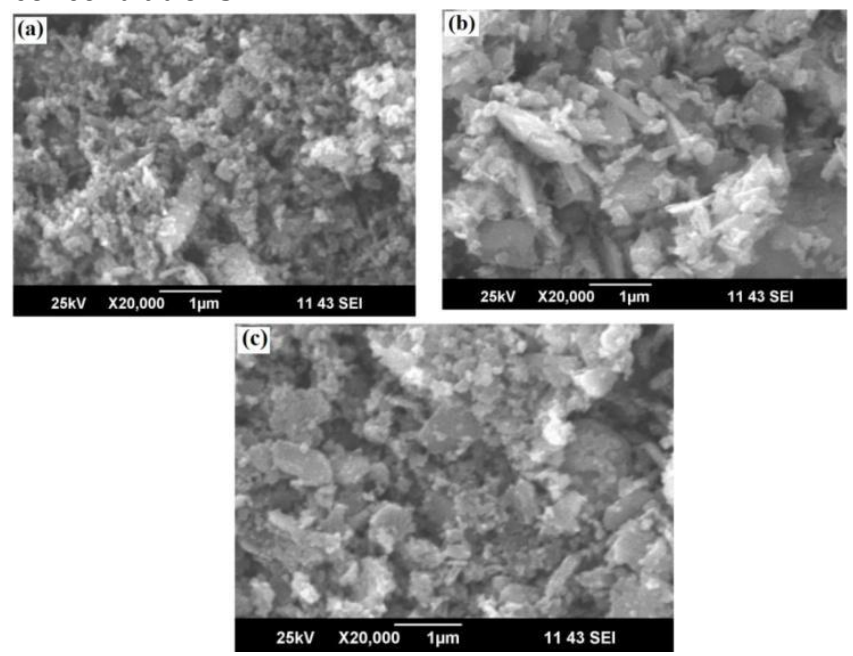

Fig. 3. SEM images of HAP nanoparticles synthesized by template directed aqueous starch method at three different concentrations (a) 0.01 wt.\% (b) 0.03 wt.\% (c) 0.05 wt.\%. 
When the concentration of the starch was 0.01 wt. \% for the synthesis of HAP, the micrograph shown in Fig. 3(a) depicts nanosized discrete particles with uniform in size distribution without any agglomeration. On increasing $t$ he starch concentration to 0.03 wt. \% (Fig. 3(b)) the particles seems feather like shape but the size is somewhat higher than previous. On further increasing of the starch addition to $0.05 \mathrm{wt}$ \% (Fig. 3(c)), the particles is in the nanometer range but unclear discrete manner were seen. Some agglomerated particles are also found in this image. Hence the optimum concentration was found to be $0.01 \mathrm{wt} \%$.

\section{CONCLUSIONS}

Hydroxyapatite nanoparticles were successfully synthesized by template directed method using aqueous starch. The FT-IR results have shown that the obtained powders were free from any organic impurities. As the addition of starch is increased, the crystallinity of the particles increased as evident from the XRD. The SEM result evident the formation of discrete spherical nanoparticles with reduced size and uniform throughout was obtained when the starch concentration was lower. The size of the particles was seen in the range around $\sim 60 \mathrm{~nm}$. Hence the developed protocol will be a convenient one as it works without complicated apparatus and chemicals. The HAP nanoparticles synthesized by this method can serve as a good biomaterial for various biomedical applications.

\section{ACKNOWLEDGEMENT}

The author acknowledges the financial support from UGC, Hyderabad, India in the form of minor research project.

\section{REFERENCES}

Bezzi, G., G. Celotti, E. Landi, T.M.G. La Torretta, I. Sopran and A. Tampieri, (2003). A novel sol-gel technique for hydroxyapatite preparation. Mater. Chem. Phys. 78: 816-824.

Dorozhkin, S.V. and M. Epple, (2002). Biological and medical significance of calcium phosphates. Angew. Chem., Int. Ed. Engl. 41(17): 3130-3146.

Gopi, D., J. Indira, L. Kavitha, M. Sekar and U. Kamachi Mudali, (2012). Synthesis of hydroxyapatite nanoparticles by a novel ultrasonic assisted with mixed hollow sphere template method. Spectrochimica Acta Part A.93:131-134.

Gopi, D., J. Indira, L. Kavitha, S. Kannan and J.M.F. Ferreira, (2010). Spectroscopic characterization of nanohydroxyapatite synthesized by molten salt method. Spectrochimica Acta Part A. 77A(2): 545-547.

Hench, L.L. (1991). Bioceramics - From concept to clinic. J. Am. Ceram. Soc. 74(7): 1487-1510.

Hench, L.L. (1998). Bioceramics. J. Am. Ceram. Soc. 81(7): 1705-1728.

Hwang, K. and Y. Lim, (1999). Chemical and structural changes of hydroxyapatite films by using a sol-gel method. Surf. Coat. Technol. 115: $172-175$.

Liou, S.C., S.Y. Chen and D.M. Liu, (2003). Synthesis and characterization of nanocomposite with controlled aspect ratios. Biomaterials 24(22): 3981-3988.

Raman, R.E., W.L. Suchanek, K. Byrappa, C.W. Chen, P. Shuk and C.S. Oakes, (2002). Solution synthesis of hydroxyapatite designer particulates. Solid State Ionics. 151(1-4): 393-402.

Yan, L., L.Y.D. Li, Z.X. Deng, J. Zhuang and X.M. Sun, (2001). Surfactant-assisted hydrothermal synthesis of hydroxyapatite nanorods. Int. J. Inorg. Mater. 3: 633-637. 